\title{
CHITOSAN MICROSPHERES FOR THE DELIVERY OF CHEMOTHERAPEUTIC AGENTS: PACLITAXEL AS A MODEL
}

\author{
BASMA YAHYA AL-NAJJAR, SAAD ABDULRAHMAN HUSSAIN*
}

Department of Pharmaceutics, Faculty of Pharmacy, Alrafidain University College, Baghdad, Iraq. Email: saad_alzaidi@yahoo.com

Received: 29 March 2017, Revised and Accepted: 28 April 2017

\section{ABSTRACT}

Chitosan has unique physicochemical and biological features that suggest it as a good candidate for the development of safe and effective drug delivery systems. Linking drug molecules with chitosan through a functional spacer enables formulation of prodrugs that have appropriate pharmacological activities at specific desired sites. The development of formulations of targeted delivery systems for the chemotherapeutic agents, especially those with unfavorable pharmacokinetic features, like paclitaxel (PTX), can potentially alleviate the systemic cytotoxicity as well as directing therapy to the specific lesions. The main aim of this literature review is to critically evaluate the use of chitosan microspheres as a drug delivery system to enhance PTX distribution and efficacy in specific targeted sites.

Keywords: Chitosan, Paclitaxel, Microspheres, Drug delivery.

(c) 2017 The Authors. Published by Innovare Academic Sciences Pvt Ltd. This is an open access article under the CC BY license (http://creativecommons. org/licenses/by/4. 0/) DOI: http://dx.doi.org/10.22159/ajpcr.2017.v10i8.18765

\section{INTRODUCTION}

One of the most significant current diseases today is cancer. It is a leading cause of death worldwide [1]. Many strategies are committed to cure cancer including chemotherapy, surgery, and radiotherapy. However, these therapies have various types serious adverse effects such as hair loss, nausea, vomiting, fatigue, sleep problems, loss of appetite, and anemia [2,3]. During the clinical phase of the disease, most of the approved drugs failed to meet the targeted clinical responses, mostly due to inability to accumulate at the target site of action, where significant amounts distributed in normal tissues or organs rather than the diseased one; this will be accompanied with emergence of severe side effects [4]. In this regard, the most effective approach to avoid this problem is through utilizing the targeted drug delivery systems, where the active moieties were released and trapped within the targeted site of action. This approach may fulfill the criteria of patient compliance and increase the efficacy of the active agents through improving their pharmacokinetic properties [5,6]. In each drug delivery system, there are three major components: An active ingredient, targeting chromophore, and carrier system. The active drug could be incorporated either by passive absorption or chemical conjugation into the carrier system. Meanwhile, the choice of a proper carrier molecule represents a significant importance due to its influence on both the pharmacokinetic and pharmacodynamic properties of the active agent [7]. Novel polymeric drug delivery systems were incorporated as a novel approach for dispensing chemotherapeutic agents and have many advantages including availability of various biocompatible polymers that enables formulation of many pharmaceutical dosage forms that utilize polymerbased drug carriers, such as microspheres, hydrogels, micelles, and nanosystems $[8,9]$. The conventional formulations of chemotherapeutic agents have inherited critical drawbacks that include high toxicity rates due to indiscriminate distribution toward normal and diseased cells after systemic administration. Moreover, poor aqueous solubility of most anticancer agents needs to use organic solvents during their formulation, with associated rise of undesirable effects such as vascular irritation and respiratory distress $[10,11]$. Therefore, utilizing an effective carrier system that can be loaded with large amounts of the active drug and specifically targeting cancer cells is considered as an interesting idea for successful cancer treatment.

A wide range of chemotherapeutic agents has been formulated using the approach of loading in a carrier system for many reasons. Chitosan- loaded drugs represent the bases of delivering hydrophobic small molecular drugs to a specific site of action [12]; this type of delivery systems includes a water-soluble polymer conjugated to the active drug through a biodegradable spacer, which is stable at circulation while cleaved at the targeted cell spontaneously or by enzymatic catalysis. Based on this concept, many delivery systems are formulated and approved for clinical use. There are many examples for this system among them is gemcitabine-loaded chitosan microspheres, where the carrier system improves cytotoxicity of the active agent [13]. Similarly, nanoparticles of gemcitabine were prepared to utilize chitosan polymer, and evaluation of this formula showed that gemcitabine-loaded particles demonstrated optimum size and maximum entrapment properties [14]. Moreover, the recently prepared methotrexate (MTX)loaded chitosan-based microspheres proved to be a promising drug delivery system that improves inject ability and compatibility, in addition to prolongation drug releasing time of injectable MTX [15]. Meanwhile, the magnetic chitosan-MTX microspheres prepared by suspension cross-linking technique demonstrated great potential application in magnetic targeting drug delivery technology [16]. Microspheres of chitosan cross-linked with polyethylene glycol were prepared by emulsion cross-linking and found to slow the release of 5 -fluorouracil up to $24 \mathrm{hr}$ to colonic region and increased its anticancer activity [17]. Furthermore, various formulations of chitosan-based models loaded with doxorubicin and cisplatin were prepared and evaluated, and the results demonstrated promising features for these newly developed formulations to improve efficacy and safety of these cytotoxic agents [18-20].

At present, chitosan-based drug delivery systems are widely utilized to target specific sites within the gastrointestinal (GI) tract since this approach can protect the highly sensitive active moieties from the unfavored conditions of the upper GI compartment. This approach enables the release of the active agent at specific sites (e.g., the colon), probably through hydrolysis of the glycosidic linkage with chitosan by the microflora [21]. In this regard, among the most investigated chemotherapeutic agents is paclitaxel (PTX), and many evidence showed that cellular association and cytotoxicity of PTX were significantly enhanced when administered as chitosan-loaded formula [22]. However, despite its many clinical advantages, PTX suffers from many limitations including low bioavailability due to its poor solubility [23]. Recently, scientists have shown an increased interest in 
formulating low therapeutic index drugs (e.g., PTX) in smart delivery systems, such as microspheres, to overcome such limitations and to avoid the fluctuations in plasma concentration of the drug, which lead to over or under medication [24]. Smart delivery systems are designed for the aim of providing safer and more efficient ways to deliver drugs with minimum adverse effects and/or enhanced therapeutic efficacy of the drug. These new drug delivery systems have become very important and might be more important than the drug itself. As a drug carrier, chitosan has been widely used as a drug delivery system for different types of biologically active molecules, including peptides, genes and small molecular weight drugs [25-27]. At present, many molecular targets are discovered and well characterized for therapeutic targeting, and the majority of clinically approved drugs belongs to the low molecular weight type [28]. Accordingly, effective delivery of these drugs, especially those with low therapeutic index or wide range of toxic effects, is considered as a growing challenge for researcher in pharmaceutical industry. The main aim of this literature review is to critically testing the use of chitosan microspheres as a drug delivery system to enhance PTX distribution and efficacy in specific targeted sites.

\section{CHITOSAN MICROSPHERES}

Microspheres are one of the most advanced approaches of controlled drug delivery systems [29]. They can be defined as spherical, small, biodegradable, and biocompatible particles [30] more than $1 \mu \mathrm{m}$ $(<100 \mu \mathrm{m})$ in size [31]. Due to the microsphere's small particle size, it has large surface area leading to a higher diffusion rate and better control of drug release [31]. Furthermore, there are several types of microspheres such as polymeric microspheres [32], radioactive microspheres [33], floating microspheres [34], and bioadhesive microspheres [35]. Microspheres have different applications in medical practice. In most instances, they are utilized as carriers and target delivery systems for many drug molecules, and for slow releasing of drugs over the required period to maintain effective drug concentrations at specific biological targets. In addition, microspheres have many other applications in food, medical devices, and water purification techniques [36]. Moreover, microspheres have many applications in pharmaceuticals manufacturing. They are used for the delivery of large molecules, fragile molecules such as proteins, vaccines, antibiotics, hormones, gene therapy, and anticancer drugs. The involvement of chitosan-loaded anticancer agents to formulate new drug delivery systems can lead to novel features of the nanoparticles, which might be explained partly due to the high surface area of the active ingredients delivery over the bulk properties [37].

Chitosan is as natural, mucoadhesive, nontoxic, biodegradable [38], and biocompatible polymer $[39,40]$. It is a product of chitin deacetylation and found in the exoskeleton of crustaceans. Due to chitosan's physical and chemical properties, its microspheres have many pharmaceutical applications $[41,42]$. Chitosan microspheres are used as a drug carrier to enhance the bioavailability of various drugs to get optimum drug release at specific site in the body. Furthermore, chitosan microspheres can be used in the formulation of either parenteral or oral drug delivery systems. Moreover, chitosan and its derivatives have the ability to be easily cross-linked with other molecules to synthesize fine particulates that can be utilized as effective drug carrier systems [43]. The process involves covalent binding of chitosan side chains with many functional cross-linking molecules, including glutaraldehyde and polyethylene glycol $[44,45]$.

There are various advantages for the chitosan microspheres, including controlled drug release pattern that may be either constant or pulsatile [46], decrease the toxicity of drugs, overcome the problems associated with hydrophilic or hydrophobic drugs, and better patient compliance. This type of formulation can be used as injectable sustained drug delivery system and help the patients to avoid frequent doses [30]; it also improves drug absorption due to the increased in vitro adhesion [38]. Furthermore, chitosan microspheres are used to enhance drug delivery through the blood-brain barrier [47], where Hassan and Gallo in 1993 showed that the bioavailability of oxantrazole-loaded chitosan microspheres could be enhanced in the brain tissue [48]. On the other hand, microspheres have a few disadvantages. They are hard to maintain the effectiveness of the drug during the production process, it is difficult to produce large quantities and expensive to produce [30].

\section{CHITOSAN MICROSPHERES FOR THE DRUG DELIVERY OF PTX}

PTX is a natural and hydrophobic anticancer agent. It has been discovered by the National Cancer Institute in 1960 [1]. It is extracted from the bark of the pacific yew tree Taxus brevifolia [49]. PTX is an influential antitumor drug against ovarian cancer, breast cancer, head and neck cancer, lung cancer [50], leukemias, melanoma, and esophageal adenocarcinoma [51,52]. Although PTX is a highly cytotoxic drug, it has limited clinical applications because of low aqueous solubility $(<1 \mu \mathrm{g} / \mathrm{ml})$ [23]. The solubility problem is due to inherited physicochemical properties. PTX structure consists of a fused ring system and many hydrophobic substituents [53], and there are no functional ionizable groups. However, PTX is available as sterile non-aqueous solutions consist of Cremophore and ethanol [54]. Unfortunately, this formulation has been observed to induce many adverse effects such as hypersensitivity and neurotoxicity [55]. Due to the problems of poor solubility and adverse effects associated with the available PTX formulation, scientists are trying to formulate it in many alternative and effective drug delivery systems, such as cyclodextrin [56,57], dendrimers [58], nanoparticles [59], prodrugs [60,61], liposomes [62], 2-methacryloyloxyethl phosphorylcholine polymers [63], and microspheres $[64,65]$. Moreover, a study performed by Mita et al. involved a new drug delivery system devoid of cremophore and composed of porous PTX microspheres [66].

In addition to chitosan loaded PTX microspheres formulation, PTX was incorporated in many other forms of chitosan-based delivery system formulations. The technique of loading PTX on chitosan involves physical complex formation rather than change of the drug loading on the nanoparticles. This approach provides most selective and safe delivery system for PTX [67]. A thermos-gelling solution of chitosan$\beta$-glycerophosphate salt and PTX was used as a local delivery system to prevent local recurrence of malignant tumors, without associated inflation and hyperthermia reported with parenteral formulation [68]. Furthermore, a hydrogel formula of PTX-loaded chitosan effectively decreases the number of CD34-positive vessels in subcutaneous 3LL tumors, indicating a strong inhibition of angiogenesis and tumor growth [69]. A PTX-loaded chitosan as thermos-sensitive hydrogel, which was modified by glutaraldehyde and polyvinyl alcohol for intratumoral delivery, was found as a promising approach to achieve effective sustained release and enhanced chemotherapeutic activity through in situ tumor injectable administration [70]. Recently, a new type of chitosan-based binary-copolymer systems containing PTXloaded micelles and siRNA-loaded LDL could directly deliver siRNA and PTX to cancer cells, offering promising chances to overcome the problem of multidrug resistance in cancer cells [71]. Moreover, parenteral nanocomposite hydrogel containing PEGylated gold nanorods and PTX-loaded chitosan was developed to improve local tumor control and found to have superior effects on preventing tumor recurrence and improving survival in the HepS-bearing mice, compared with photo-thermal therapy alone [72]. It has been also shown that PTX incorporated into polymeric micelles based on tocopherol succinate-chitosan-polyethylene glycol-folic acid micelle is a potential drug delivery system of PTX for the effective treatment of the tumor and reduction of systemic toxicity, which may provide a useful alternative formula for intravenous administration of PTX [73]. Among the different studied drug carriers, microparticles particularly chitosan-based microparticles have been used to enhance the solubility of anticancer drugs such as oxantrazole [74], PTX [65], and MTX [75].

Microspheres have been approved to enhance the solubility, dissolution rate, and bioavailability of PTX $[65,76]$. Al-shdefat et al. demonstrated 
that chitosan microspheres influence the dissolution of PTX [65]. The presence of PTX in porous, amorphous form and small particle size in the microspheres further enhances its dissolution. The high zeta potential values of the particles allow maintenance of their low particle sizes, which prevent aggregation of the microparticles and increase PTX dissolution rate. It has been previously reported that the medium molecular weight of chitosan formulae exhibited the highest PTX dissolution rate because of the highest aqueous perfusable microspheres $[65,77]$, where $90 \%$ of the drug could be released from the nanosuspension.

In addition to enhancing the solubility and dissolution rate of PTX, chitosan microspheres have also been demonstrated to entrap high percentages of PTX. Kollipara et al. have measured the entrapment efficiency of PTX in chitosan microspheres utilizing high-performance liquid chromatography. Different formulas have demonstrated different entrapment efficiencies of PTX depending on the molecular weight of chitosan and $\mathrm{pH}$ of the formulation and it was between 21-83.7\% [78]. Moderate acidic medium (pH 3-5) is the ideal crosslinking media to get the highest entrapment efficiencies of PTX (83\%, 83.7\%, and $73.7 \%)[65,79]$. Moreover, it has been reported that the highest entrapment efficiencies of PTX when incorporated in poly(lactic acid)-poly(ethylene glycol)-poly(lactic acid) (PLA-PEGPLA) microspheres were 81.55, 81.53, and 73.93\%. Furthermore, the reported PTX entrapment efficiencies in microspheres were higher than the entrapment efficiencies in other types of carrier systems such as liposomes $(61.02 \pm 1.61 \%)$ [62] and gelatin nanoparticles $(25 \%)[80]$

In this regard, many investigators have also studied the release pattern of PTX from chitosan microspheres. They found that PTX release pattern was rapid in the first hour; then, the cumulative release amount was increased within $24 \mathrm{hrs}$ [65], and the drug release pattern varies between different microspheres formulae. They specifically considered the microspheres' particle size and the amorphous form of PTX as the two major factors that influence both dissolution and release pattern of PTX. Thus, the smaller particle sizes exhibited the highest drug release profiles. Moreover, the viscosity of chitosan could affect the release pattern of the drug with consequent impact on the biological activity $[81,82]$. It has been demonstrated also that increasing the film thickness of chitosan decreases the release pattern of a drug [83], in addition to the influence of the medium condition that affects the release pattern of PTX from microspheres [84]. Cho et al. studied the in vitro drug release pattern of PTX loaded chitosan microspheres in sodium salicylate as a medium and found that the solubility of PTX could be enhanced by 100 times in sodium salicylate without damaging the micellar structure of polymeric micelle drug delivery [84]. Although Al-shdefat et al. study involved PTX loaded chitosan microspheres and considered the only study in this regard, there are various studies which involve using other polymers with microspheres such as chitin microparticles [65,85], chitosan poly(lactic-co-glycolic acid) (PLGA) microparticles [22], PLA-PEG-PLA microspheres [79], PLA and ethylene vinyl acetate copolymer [63], and PTX-loaded PLGA microspheres containing isopropyl myristate [86].

\section{CONCLUSION}

This critical review has explained the delivery of PTX loaded chitosan microspheres. This drug delivery system has been designed to enhance PTX delivery at the tumor site and to reduce its side effects. The most significant finding in this review is that PTX limitations can be effectively avoided through incorporation in chitosan microspheres; thus, improving its solubility, dissolution rate, entrapment efficiency, and its release pattern. Accordingly, chitosan microspheres represent a promising future for the delivery of PTX.

\section{ACKNOWLEDGMENT}

The authors thank Alrafidain University College for encouragement and support.

\section{REFERENCES}

1. Panchagnula R. Pharmaceutical aspects of paclitaxel. Int J Pharm 1998;172:1-15.

2. Walbert T, Gilbert MR, Groves MD, Puduvalli VK, Yung WK, Conrad CA, et al. Combination of 6-thioguanine, capecitabine, and celecoxib with temozolomide or lomustine for recurrent high-grade glioma. J Neurooncol 2011;102(2):273-80.

3. Chang CH, Horton J, Schoenfeld D, Salazer O, Perez-Tamayo R, Kramer S, et al. Comparison of postoperative radiotherapy and combined postoperative radiotherapy and chemotherapy in the multidisciplinary management of malignant gliomas. A joint Radiation Therapy Oncology Group and Eastern Cooperative Oncology Group study. Cancer 1983;52(6):997-1007.

4. Patel MP, Patel RR, Patel JK. Chitosan mediated targeted drug delivery system: A review. J Pharm Pharm Sci 2010;13(4):536-57.

5. Langer R. Drug delivery and targeting. Nature 1998;392:5-10

6. DiMasi JA, Hansen RW, Grabowski HG. The price of innovation: New estimates of drug development costs. J Health Econ 2003;22(2):151-85.

7. Weng L, Rostamzadeh P, Nooryshokry N, Le HC, Golzarian J. In vitro and in vivo evaluation of biodegradable embolic microspheres with tunable anticancer drug release. Acta Biomater 2013;9(6):6823-33.

8. Makadia HK, Siegel SJ. Poly lactic-co-glycolic acid (PLGA) as biodegradable controlled drug delivery carrier. Polymers (Basel) 2011;3(3):1377-97.

9. Acharya G, Shin CS, Vedantham K, McDermott M, Rish T, Hansen K, et al. A study of drug release from homogeneous PLGA microstructures. J Control Release 2010;146(2):201-6.

10. Torchilin VP. Targeted polymeric micelles for delivery of poorly soluble drugs. Cell Mol Life Sci 2004;61(19-20):2549-59.

11. Wolinsky JB, Colson YL, Grinstaff MW. Local drug delivery strategies for cancer treatment: Gels, nanoparticles, polymeric films, rods, and wafers. J Control Release 2012;159(1):14-26.

12. Ringsdorf H. Structure and properties of pharmacologically active polymers. J Polym Sci Polym Symp 1975;51:135-53.

13. Ventura CA, Cannavà C, Stancanelli R, Paolino D, Cosco D, La Mantia A, et al. Gemcitabine-loaded chitosan microspheres. Characterization and biological in vitro evaluation. Biomed Microdevices 2011;13(5):799-807.

14. Gupta V, Karar PK. Optimization of process variables for the preparation of chitosan-alginate nanoparticles. Int J Pharm Pharm Sci 2011;3(2):78-80.

15. Dang Q, Liu C, Wang Y, Yan J, Wan H, Fan B. Characterization and biocompatibility of injectable microspheres-loaded hydrogel for methotrexate delivery. Carbohydr Polym 2016;136:516-26.

16. Zhang X, Chen F, Ni J. A novel method to prepare magnetite chitosan microspheres conjugated with methotrexate (MTX) for the controlled release of MTX as a magnetic targeting drug delivery system. Drug Deliv 2009;16(5):280-8.

17. Ganguly K, Kulkarni AR, Aminabhavi TM. In vitro cytotoxicity and in vivo efficacy of 5-fluorouracil-loaded enteric-coated PEG-crosslinked chitosan microspheres in colorectal cancer therapy in rats. Drug Deliv 2015;1:1-14.

18. Park JM, Lee SY, Lee GH, Chung EY, Chang KM, Kwak BK, et al. Design and characterization of doxorubicin-releasing chitosan microspheres for anti-cancer chemo-embolization. J Microencapsul 2012;29(7):695-705

19. Akbuga J, Bergisadi N. Effect of formulation variables on cisplatin loaded chitosan microsphere properties. J Microencapsul 1999;16(6):697-703.

20. Wang YM, Sato H, Adachi I, Horikoshi I. Optimization of the formulation design of chitosan microspheres containing cisplatin. J Pharm Sci 1996;85(11):1204-10.

21. Hejazi R, Amiji M. Chitosan-based gastrointestinal delivery systems. J Control Release 2003;89:151-65.

22. Chakravarthi SS, Robinson DH. Enhanced cellular association of paclitaxel delivered in chitosan-PLGA particles. Int $\mathrm{J}$ Pharm 2011;409(1-2):111-20.

23. Martin V. Overview of paclitaxel (TAXOL). Semin Oncol Nurs 1993;9 4 Suppl 2:2-5.

24. Vilos C, Velasquez LA. Therapeutic strategies based on polymeric microparticles. J Biomed Biotechnol 2012;2012:672760.

25. Kim JH, Kim YS, Park K, Lee S, Nam HY, Min KH, et al. Antitumor efficacy of cisplatin-loaded glycol chitosan nanoparticles in tumorbearing mice. J Control Release 2008;127(1):41-9.

26. Yoo HS, Lee JE, Chung H, Kwon IC, Jeong SY. Self-assembled nanoparticles containing hydrophobically modified glycol chitosan for 
gene delivery. J Control Release 2005;103(1):235-43.

27. Amidi M, Romeijn SG, Borchard G, Junginger HE, Hennink WE, Jiskoot W. Preparation and characterization of protein-loaded $\mathrm{N}$-trimethyl chitosan nanoparticles as nasal delivery system. J Control Release 2006;111(1-2):107-16.

28. Overington JP, Al-Lazikani B, Hopkins AL. How many drug targets are there? Nat Rev Drug Discov 2006;5(12):993-6.

29. Mathiowitz E, Jacob JS, Jong YS, Carino GP, Chickering DE, Chaturvedi $\mathrm{P}$, et al. Biologically erodable microspheres as potential oral drug delivery systems. Nature 1997;386(6623):410-4.

30. Kim K, Pack DW. Microspheres for the delivery. Biomembr Biomed Nanotechnol 2006;1:19-50.

31. Kumar SK, Reddy JP, Sekhar CK. A review on microsphere for novel drug delivery system. J Pharm Res 2012;5(1):420-4.

32. Ando S, Putnam D, Pack DW, Langer R. PLGA microspheres containing plasmid DNA: Preservation of supercoiled DNA via cryopreparation and carbohydrate stabilization. J Pharm Sci 1999;88(1):126-30.

33. Amsden BG, Goosen M. An examination of the factors affecting the size, distribution, and release characteristics of polymer microbeads made using electrostatics. J Control Release 1997:43:183-96.

34. Leon L, Herbert AL, Joseph LK. The Theory and Practice of Industrial Pharmacy. $3^{\text {rd }}$ ed. UK: Varghese Publishing House; 1990. p. 414-5.

35. Thanou M, Nihot MT, Jansen M, Verhoef JC, Junginger HE. MonoN-carboxymethyl chitosan (MCC), a polyampholytic chitosan derivative, enhances the intestinal absorption of low molecular weight heparin across intestinal epithelia in vitro and in vivo. J Pharm Sci 2001;90(1):38-46

36. Nithyashanthi C, Gupta R, Mahato AK. Traditional and emerging applications of microspheres. Int J PharmTech Res 2010;2(1):675-81.

37. Hemant KS, Raizaday A, Sivadasu P, Hemanth-Kumar S. Cancer nanotechnology: Nanoparticulate drug delivery for the treatment of cancer Int J Pharm Pharm Sci 2015;7(3):40-6.

38. Bansal V, Sharma KP, Sharma N, Pal OP, Malviya R. Application of chitosan and chitosan derivatives in drug delivery. Adv Biol Res 2011;5(1):28-37

39. Hirano S, Nagano N. Effects of chitosan, pectic acid, lysozyme, and chitinase on the growth of several phytopathogens. Agric Biol Chem 1989;53:3065-6.

40. Lehr CM, Bouwstra JA, Schacht EH, Junginger HE. In vitro evaluation of mucoadhesive properties of chitosan and some other natural polymers. Int J Pharm 1992;78(1):43-8.

41. Sanford PA. Chitosan: Commercial uses and potential applications. In: Skjak G, Anthonsen T, Sanford P, editors. Chitin and Chitosan: Sources, Chemistry, Biochemistry, Physical Properties and Applications. London, UK: Elsevier; 1989. p. 51-69.

42. Kas HS. Chitosan: Properties, preparations and application to microparticulate systems. J Microencapsul 1997;14(6):689-711.

43. Prabaharan M, Mano JF. Chitosan-based particles as controlled drug delivery systems. Drug Deliv 2005;12:41-57.

44. Goldberg M, Langer R, Jia X. Nanostructured materials for applications in drug delivery and tissue engineering. J Biomater Sci Polym Ed 2007;18(3):241-68.

45. Bodnar M, Hartmann JF, Borbely J. Preparation and characterization of chitosan-based nanoparticles. Biomacromolecules 2005;6(5):2521-7.

46. Genta I, Costantini M, Asti A, Conti B, Montanari L. Influence of glutaraldehyde on drug release and mucoadhesive properties of chitosan microspheres. Carbohydr Polym 1998;36(2-3):81-8

47. Soppimath KS, Aminabhavi TM, Kulkarni AR, Rudzinski WE. Biodegradable polymeric nanoparticles as drug delivery devices. J Control Release 2001;70(1-2):1-20.

48. Hassan EE, Gallo JM. Targeting anticancer drugs to the brain. I: Enhanced brain delivery of oxantrazole following administration in magnetic cationic microspheres. J Drug Target 1993;1(1):7-14.

49. Wani MC, Taylor HL, Wall ME, Coeggon P, McPhail AT. The discovery of camptothecin and taxol. J Am Chem Soc 1971;93(9):2325-7.

50. Rowinsky EK, Cazenave LA, Donehower RC. Taxol: A novel investigational antimicrotubule agent. J Natl Cancer Inst 1990;82(15):1247-59.

51. Manfredi JJ, Horwitz SB. Taxol: An antimitotic agent with a new mechanism of action. Pharmacol Ther 1984;25(1):83-125.

52. Spencer CM, Faulds D. Paclitaxel. A review of its pharmacodynamic and pharmacokinetic properties and therapeutic potential in the treatment of cancer. Drugs 1994;48(5):794-847.

53. Straubinger RM. Biopharmaceutics of paclitaxel (taxol): Formulation, activity and pharmacokinetics. In: Suffness M, editor. Science and Applications. Boca Raton: CRC Press; 1996. p. 237-58.

54. Barbara E. The Effect of Excipients on Pharmacokinetic Parameters of
Parenteral Drugs. 2005, PhD Thesis, University of Basel, Faculty of Science.

55. Gelderblom H, Verweij J, Nooter K, Sparreboom A. Cremophor EL: The drawbacks and advantages of vehicle selection for drug formulation. Eur J Cancer 2001;37(13):1590-8.

56. Londhe V, Nagarsenker M. Comparison between hydroxypropyl- $\beta$ cyclodextrin and polyvinylpyrrolidone as carriers for carbamazepine solid dispersions. Indian J Pharm Sci 1999;61:237-40

57. Bettinetti G, Gazzaniga A, Mura P, Giordano F, Setti M. Thermal behavior and dissolution properties of naproxen in combinations with chemically modified beta-cyclodextrins. Drug Dev Ind Pharm 1992;18:39-53

58. Ooya T, Lee J, Park K. Effects of ethylene glycol-based graft, starshaped, and dendritic polymers on solubilization and controlled release of paclitaxel. J Control Release 2003;93(2):121-7.

59. Lv PP, Wei W, Yue H, Wang LY, Ma GH. Porous quaternized chitosan nanoparticles containing paclitaxel nanocrystals improved therapeutic efficacy in non-small-cell lung cancer after oral administration. Biomacromolecules 2011;12(12):4230-9.

60. Greenwald RB, Pendri A, Bolikal D, Gilbert CW. Highly water soluble taxol derivatives: 2' polyethylene glycol esters as potential prodrugs. Bioorg Med Chem Lett 1994;4:2465-70.

61. Greenwald RB, Gilbert CW, Pendri A, Conover CD, Xia J, Martinez A. Drug delivery systems: Water soluble taxol 2'-poly(ethylene glycol) ester prodrugs-design and in vivo effectiveness. J Med Chem 1996;39(2):424-31.

62. Yang T, Cui FD, Choi MK, Cho JW, Chung SJ, Shim CK, et al. Enhanced solubility and stability of PEGylated liposomal paclitaxel: In vitro and in vivo evaluation. Int J Pharm 2007;338(1-2):317-26.

63. Konno T, Watanabe J, Ishihara K. Enhanced solubility of paclitaxel using water-soluble and biocompatible 2-methacryloyloxyethyl phosphorylcholine polymers. J Biomed Mater Res A 2003;65(2):209-14.

64. Dordunoo SK, Jackson JK, Arsenault LA, Oktaba AM, Hunter WL, Burt HM. Taxol encapsulation in poly(epsilon-caprolactone) microspheres. Cancer Chemother Pharmacol 1995;36(4):279-82.

65. Al-shdefat R, Yassin AB, Anwer K, Alsarra I. Preparation and characterization of biodegradable paclitaxel loaded chitosan microparticles. Dig J Nanomater Biostruct 2012;7(3):1139-47.

66. Mita AC, Olszanski AJ, Walovitch RC, Perez RP, MacKay K, Tuck DP, et al. Phase I and pharmacokinetic study of AI-850, a novel microparticle hydrophobic drug delivery system for paclitaxel. Clin Cancer Res 2007;13(11):3293-301.

67. Abdulbaqi MR, Maraie NK, Dawood AH. Loading of clarithromycin and paclitaxel on synthesized CDS/NIO nanoparticles as promising nanocarriers. Int J Pharm Pharm Sci 2016;8(5):322-33.

68. Ruel-Gariepy E, Shive M, Bichara A, Berrada M, Le Garrec D, Chenite A, et al. A thermos-sensitive chitosan-based hydrogel for the local delivery of paclitaxel. Eur J Pharm Biopharm 2004;57:53-63.

69. Obara K, Ishihara M, Ozeki Y, Ishizuka T, Hayashi T, Nakamura S, et al. Controlled release of paclitaxel from photo cross-linked chitosan hydrogels and its subsequent effect on subcutaneous tumor growth in mice. J Control Release 2005;110(1):79-89.

70. Jiang Y, Meng X, Wu Z, Qi X. Modified chitosan thermosensitive hydrogel enables sustained and efficient anti-tumor therapy via intratumoral injection. Carbohydr Polym 2016;144:245-53.

71. Yang SD, Zhu WJ, Zhu QL, Chen WL, Ren ZX, Li F, et al. Binarycopolymer system based on low-density lipoprotein-coupled $\mathrm{N}$-succinyl chitosan lipoic acid micelles for co-delivery MDR1 siRNA and paclitaxel, enhances antitumor effects via reducing drug. J Biomed Mater Res B Appl Biomater 2016. DOI: 10.1002/jbm.b.33636.

72. Zhang N, Xu X, Zhang X, Qu D, Xue L, Mo R, et al. Nanocomposite hydrogel incorporating gold nanorods and paclitaxel-loaded chitosan micelles for combination photothermal-chemotherapy. Int J Pharm 2016;497(1-2):210-21.

73. Rezazadeh M, Emami J, Hasanzadeh F, Sadeghi H, Minaiyan M, Mostafavi A, et al. In vivo pharmacokinetics, biodistribution and antitumor effect of paclitaxel-loaded targeted chitosan-based polymeric micelle. Drug Deliv 2016;23(5):1707-17.

74. Hassan EE, Parish RC, Gallo JM. Optimized formulation of magnetic chitosan microspheres containing the anticancer agent, oxantrazole. Pharm Res 1992;9(3):390-7.

75. Singh UV, Udupa N. Methotrexate loaded chitosan and chitin microspheres - In vitro characterization and pharmacokinetics in mice bearing Ehrlich ascites carcinoma. J Microencapsul 1998;15(5):581-94.

76. Terwogt JM, Nuijen B, Huinink WW, Beijnen JH. Alternative formulations of paclitaxel. Cancer Treat Rev 1997;23(2):87-95.

77. Wang Y, Li X, Wang L, Xu Y, Cheng X, Wei P. Formulation and 
pharmacokinetic evaluation of a paclitaxel nanosuspension for intravenous delivery. Int J Nanomedicine 2011;6:1497-507.

78. Kollipara S, Bende G, Saha RN. Rapid and sensitive liquid chromatographic method for determination of paclitaxel from parenteral formulation and nanoparticles. Indian J Pharm Sci 2010;72(4):465-70.

79. Ruan G, Feng SS. Preparation and characterization of poly(lactic acid)poly(ethylene glycol)-poly(lactic acid) (PLA-PEG-PLA) microspheres for controlled release of paclitaxel. Biomaterials 2003;24:5037-44.

80. Chanchall A, Husain SA, Verma AK. In-vitro physico-chemical evaluation of biopolymeric nanoparticles. J Pharm Res 2012;5(8):4042-4.

81. Chiou SH, Wu WT, Huang YY, Chung TW. Effects of the characteristics of chitosan on controlling drug release of chitosan coated PLLA microspheres. J Microencapsul 2001;18(5):613-25.

82. Mi FL, Her NL, Kuan CY, Wong TB, Shyu SS. Chitosan tablets for controlled release of theophylline: Effect of polymer-drug wet or dry blending and anionic-cationic interpolymer complex. J Appl Polym Sci 1997;66:2495-505.

83. Singh DK, Ray AR. Controlled release of glucose through modified chitosan membranes. J Membr Sci 1999;155:107-12.

84. Cho YW, Lee J, Lee SC, Huh KM, Park K. Hydrotropic agents for study of in vitro paclitaxel release from polymeric micelles. J Control Release 2004;97(2):249-57.

85. Nsereko S, Amiji M. Localized delivery of paclitaxel in solid tumors from biodegradable chitin microparticle formulations. Biomaterials 2002;23(13):2723-31.

86. Wang YM, Sato H, Horikoshi I. In vitro and in vivo evaluation of taxol release from poly(lactic-co-glycolic acid) microspheres containing isopropyl myristate and degradation of the microspheres. J Control Release 1997;49:157-66. 